\title{
Citrus Flavone Tangeretin Inhibits CRPC Cell Proliferation by Regulating Cx26, AKT, and AR Signaling
}

\author{
Ningfang Zhang $\mathbb{D}^{1},{ }^{1}$ Wenqi $W u,{ }^{2,3}$ Yapeng Huang, ${ }^{3}$ Lingyue An ${ }^{D},{ }^{4}$ Zhichan He, ${ }^{4}$ \\ Zhenglin Chang, ${ }^{4}$ Zhaohui He $\mathbb{D}^{1},{ }^{1}$ and Yongchang Lai $\mathbb{D}^{1,2,4}$ \\ ${ }^{1}$ The Eighth Affiliated Hospital of Sun Yat-sen University, Shenzhen, Guangdong 518033, China \\ ${ }^{2}$ Guangdong Key Laboratory of Urology, Guangzhou 510230, China \\ ${ }^{3}$ Department of Urology, The Second Affiliated Hospital of Guangzhou Medical University, Guangzhou 510230, China \\ ${ }^{4}$ Department of Urology, Minimally Invasive Surgery Center, The First Affiliated Hospital of Guangzhou Medical University, \\ Guangzhou Urology Research Institute, Guangzhou 510230, China
}

Correspondence should be addressed to Zhaohui He; hechh9@mail.sysu.edu.cn and Yongchang Lai; laiych8@mail.sysu.edu.cn

Received 30 May 2021; Accepted 6 December 2021; Published 24 January 2022

Academic Editor: Sakthivel Muniyan

Copyright () 2022 Ningfang Zhang et al. This is an open access article distributed under the Creative Commons Attribution License, which permits unrestricted use, distribution, and reproduction in any medium, provided the original work is properly cited.

\begin{abstract}
Prostate cancer ( $\mathrm{PCa})$ progression depends on the action of androgen receptors (AR). Therefore, preventing ligand-mediated activation of AR is the first-line treatment strategy for metastatic PCa. Androgen deprivation therapy (ADT) can inhibit ligand binding to AR and alleviate PCa progression initially. However, due to the adaptation of PCa and recovery of AR signaling, castration-resistant prostate cancer (CRPC) eventually develops. Exploring novel dietary compounds that can target AR signaling appears to be a viable alternative therapeutic option for CRPC. In the present study, compounds from the citrus fruits were focused upon, which contain various flavonoid ingredients. Key components contained within orange peel, which is frequently used in traditional Chinese medicine, and downstream targets were first analyzed using network pharmacology approach. Notably, it was found that tangeretin, an active ingredient from orange peel, can significantly inhibit CRPC cell (C4-2 and Du145 cells) proliferation and migration whilst also synergistically increasing the sensitivity of CRPC cells to anti-tumor drugs sorafenib or cisplatin. Tangeretin also significantly reduced AR and AKT expressions in C4-2 cells and signal transducer and activator of transcription 3 expression in the androgen-insensitive cell line Du145. In addition, tangeretin increased the expression of both connexin26 (Cx26) and gap junction function, which may mediate the bystander effects of cisplatin or sorafenib. Taken together, the present study revealed a novel molecular mechanism by which tangeretin may inhibit the proliferation of CRPC cells, by affecting the Cx26/AKT/AR pathway, to synergistically increase the sensitivity of CRPC cells to sorafenib and cisplatin.
\end{abstract}

\section{Introduction}

Prostate cancer (PCa) progression depends on androgen receptor (AR) activity [1]. Therefore, preventing the ligand activation of ARs is one of the key treatment strategies for metastatic PCa [1]. Androgen deprivation therapy (ADT) serves to inhibit AR ligand binding and can halt PCa progression initially. However, due to the adaptation of PCa physiology and recovery of AR signaling, a large proportion of patients eventually recur and develop castration-resistant prostate cancer (CRPC) [2]. Regulation of
AR signaling during CRPC progression can be mediated by AR signaling amplification and overexpression, AR gene mutations, and AR gene splicing. In particular, splice variants of $A R$ or $A R$ mutants can contribute to resistance to $\mathrm{ADT}$, radiotherapy, or chemotherapy in patients with AR-positive PCa. Although AR inhibitors, such as enzalutamide, abiraterone, and yew alkane drugs, such as docetaxel, are drugs that are commonly available for treating CRPC, therapeutic strategies targeting the fulllength $A R, A R$ mutants, and AR-splice variants remained to be unexplored and underdeveloped [3]. 
Abnormal gap junction (GJ) function has been previously associated with the occurrence, growth, invasion, and metastasis of tumors in addition to resistance to therapy [4]. GJs are assembled by two connexins, which are then assembled into hexamers to form a hemichannel. It allows the transmission of small molecules $(<1 \mathrm{kDa})$, such as ions, metabolites, and even anti-cancer drugs [5]. GJ-mediated exchange of materials between two neighboring cells is termed "gap junctional intercellular communication" (GJIC), which may facilitate the bystander effects of anticancer drugs, such as cisplatin [6], sunitinib [7], and dioscin [8]. In addition, connexins can also regulate the occurrence and development of tumors independent of GJ formation [9]. Therefore, GJ/Cx is a potential therapeutic target against cancer metastasis and chemoresistance.

A variety of flavonoids have been reported to exert antitumor effects and can regulate the function of $\mathrm{GJ} / \mathrm{Cx}$ [10]. Among these, tangeretin has been documented to significantly reverse the impairments in GJIC function induced by tumor promoters such as 12-O-tetradecanoylphorbol-acetate (TPA) and 3,5,di-tert-butyl-4-hydroxytoluene (BHT) [11]. Tangeretin is a polymethoxy flavonoid contained within citrus fruit peels and has a molecular weight of $372.37 \mathrm{~g} / \mathrm{mol}$, which partially meets the criteria of potential candidate drugs (i.e., the molecular weight is $<500 \mathrm{~g} / \mathrm{mol}$, the number of hydrogen bond donors is $<5$, and the number of hydrogen bond receptors is $<10$ ). A number of studies have previously shown that tangeretin exerts cytotoxic effects on various cancer cell types [12] and inhibits angiogenesis and metastasis. Furthermore, tangeretin has been reported to scavenge reactive radicals [13] and alleviate inflammation [14]. Cotreatment with tangeretin was also found to not only enhance the sensitivity of hepatoma carcinoma cells, colon carcinoma cells, and ovarian cancer cells to cisplatin [15] but also alleviate kidney damage caused by cisplatin [16] and the carcinogen dimethylbutyric acid [17].

The peel of the orange is rich in polymethoxy flavonoids, whereas citrus is the dry and ripe peel of the orange fruit. Tangeretin has been found to mediate functions of sterilization [18], anti-oxidation, and tumor inhibition [19]. The citrus flavonoid compound hesperetin has been demonstrated to suppress taxane cytotoxicity in prostate cancer cells [20]. In addition, the pectin content of citrus can enhance the sensitivity of prostate cancer to radiation therapy [21]. A previous study investigated the daily fruit and vegetable intake of 142,239 men from 8 countries for 13.9 years, including 7,036 men with prostate cancer [22]. This study found that long-term citrus consumption was associated with a lower risk of prostate cancer [22]. However, for the reason that citrus and orange are rich in a variety of active compounds, the relationship between the different components in citrus and prostate cancer remains unclear. The citrus flavone tangeretin has been found to exhibit biological activities in PC-3 and LNCaP cells by targeting the phosphoinositide 3-kinase (PI3K)/protein kinase $\mathrm{B}(\mathrm{AKT}) / \mathrm{mammalian}$ target of rapamycin (mTOR) and p21 signaling pathways $[23,24]$. However, whether the active compounds contained within citrus can inhibit the proliferation of CRPC cells by regulating GJ/Cx, and AR expression remain poorly understood.

In the present study, the network pharmacology analysis was used to screen for the active components and targets within orange peel, following which a series of experiments were performed to verify the results in vitro. It was found that tangeretin can inhibit CRPC cell proliferation and increase the potency of cisplatin or sorafenib by modulating the expression of proteins in the Cx26/AKT/AR signaling pathway whilst upregulating the function of GJs.

\section{Materials and Methods}

2.1. Pharmacology Analysis and Molecular Docking. Active compounds contained within orange peel were identified using the Traditional Chinese Medicine Systems Pharmacology (TCMSP) database, SymMap database (http://www.symmap.org/), and reported literature. Following absorption, distribution, metabolism, excretion, and toxicity (ADME/T) calculations (oral bioavailability $>20 \%$, druglikeness $>0.1$, and permeability Caco- $2 \geq-0.4$ ), compounds were identified to be active and potentially effective compounds. Genes that were predicted to be targeted by orange peel compounds were obtained using the TCMSP, SymMap, and Swiss Target Prediction databases, whereas target genes associated with PCa physiology were obtained using the Online Mendelian Inheritance in Man (OMIM; omim.org/) and GeneCards databases (http://www. genecards.org/). Predicted target proteins were also obtained and converted to genes using the Uniprot database (http://www.uniprot.org/). A citrus-compound-target network was generated using the Cytoscape tool (3.6.0; https:// cytoscape.org/). The protein-protein interaction (PPI) network between the potential targets was analyzed using a search tool for retrieval of interacting genes (STRING; http://www.string-db.org) with the species limited to "Homo sapiens." Each node represents proteins produced by a single protein-coding gene locus. Gene ontology (GO) classifications and Kyoto Encyclopedia of Genes and Genomes (KEGG) pathways annotations were searched using the webbased Database for Annotation, Visualization, and Integrated Discovery (DAVID) tool (david.ncifcrf.gov/tools.jsp). The process of molecular ducking was done according to the method described by Yu et al. [25].

2.2. Materials. Tangeretin, sorafenib, and cisplatin were purchased from Selleck Chemicals (Houston, USA). Anti$\mathrm{AR}$, antiphosphorylated (p) AKT, anti-AKT, and antisignal transducer and activator of transcription 3 (STAT3) antibodies were acquired from Cell Signaling Technology (Danvers, USA). The antiactin antibody (GB11001) was purchased from Wuhan Servicebio Technology Co. Ltd. (Wuhan, Hubei, China). The anti-Cx26 antibody (bs-1715R) was obtained from BIOSS (Beijing, China).

2.3. Cell Lines and Cell Cultures. CRPC cell lines C4-2 and Du145 cells were obtained from the American Type Culture Collection (Manassas, USA). A-375 cells were purchased 
from the National Collection of Authenticated Cell Cultures (Shanghai, China). Du145 and A-375 cells were cultured in Dulbecco's Modified Eagle Medium supplemented with 10\% fetal bovine serum (FBS, Thermo Fisher Scientific, Waltham, USA), whilst C4- 2 cells were cultured in RPMI-1640 supplemented with $10 \%$ FBS. All cells were cultured at $37^{\circ} \mathrm{C}$ and $5 \% \mathrm{CO}_{2}$ in a humidified atmosphere. To construct $\mathrm{Cx} 26-$ overexpressing cell lines, a full-length Cx26 cDNA was inserted into the pCMV-MCS-3Flag vector (Beijing Ruiboxingke Biotechnology Co. Ltd., Beijing, China). The transfection procedure was performed in accordance with the instructions of the Lipofectamine ${ }^{\circledR} 3000$ transfection reagent (Thermo Fisher Scientific, Waltham, USA).

2.4. Cell Viability Test. The effect of tangeretin on the proliferation of CRPC cell lines C4-2 and Du145, either alone or in combination with sorafenib or cisplatin, was detected using the 3-(4,5-dimethylthiazol-2-yl)-5-(3-carboxymethoxyphenyl)-2-(4-sulfonphenyl)-2H-tetrazolium (MTS) assay (Beibokit, China). Briefly, after the cells were incubated with the corresponding drugs for $24 \mathrm{~h}$ in the corresponding medium, the medium was replaced with a fresh medium $(90 \mu \mathrm{l})$ containing MTS $(10 \mu \mathrm{l})$ and incubated in $37^{\circ} \mathrm{C}$ for $1-3 \mathrm{~h}$. Subsequently, optical values in each well were measured using a multimode reader (BioTek Instruments, Inc., Winooski, USA) at $490 \mathrm{~nm}$. The control group was normalized to "100," before cell viability was detected and the relative survival rates were evaluated.

2.5. Cell Scratch Test. PCa cell migration ability was detected by cell scratch test. After the formation of the cell monolayer, a sterile $200 \mu$ l yellow pipette tip was used to scratch the monolayer cells to make a straight line wound. Before the cells were incubated with a fresh cell culture medium containing $1-3 \%$ FBS (in a reduced serum condition) and tangeretin, the cells were washed with PBS three times. The nicks were then photographed using microscopy (light).

2.6. Colony Formation Assay. Cells suspension with a density of 500 cells $/ \mathrm{ml}$ was seeded into the culture dish. In total, $24 \mathrm{~h}$ later, the medium was replaced with fresh medium containing tangeretin and sorafenib. Subsequently, 10-15 days later, after the macroscopic clones were formed in the culture dish, cells were washed twice with PBS before $4 \%$ alcohol crystal violet was added to fix and stain the cells for $20 \mathrm{~min}$. The dye solution was washed away using distilled water, and the cells were air-dried. The colonies were imaged, and then the number of colonies was calculated.

2.7. Western Blotting. The protocol of the western blotting was performed according to that described in a previous study [26]. Briefly, after the cells were incubated with the tangeretin and sorafenib for $24 \mathrm{~h}$, the total protein of cells was lysed using RIPA buffer (Thermo Fisher Scientific, Waltham, USA). After the samples were quantified and configured to the same concentration, each lane was loaded with $20 \mu \mathrm{g}$ total protein, and they were separated by $10 \%$
SDS-PAGE; the protein samples were transferred onto polyvinylidene fluoride (PVDF) membranes and blocked by 5\% skimmed milk. Anti-Stat3 (1:1,000), pAKT (1:1,000), AKT (1:1,000), Cx26 (1:1,000), and AR (1:1,000) primary antibodies together with their corresponding secondary antibodies (1:2,000, CellSignaling Technology\#7074, USA and 1:2,000, CellSignaling Technology\#7076, USA) were used to incubate the PVDF membranes. After the PVDF membranes were scanned using the two-color infrared fluorescence protein analysis system (Li-cor Image Studio Ver5.2 software; LI-COR Biosciences, Lincoln, USA), the scanned image was exported before the grayscales were analyzed using the Image J software (National Institutes of Health).

2.8. Parachute Dye-Coupling Assay. This assay was used to evaluate the gap junction intracellular communication (GJIC) function as described previously. Donor and receiver cells were first grown to confluence [27]. "Donor cells" were then labeled with $0.5 \mu \mathrm{mol} / 1$ Calcein-AM (Thermo Fisher Scientific, Waltham, USA), which is a membrane dye that can spread to coupled cells. The donor cells were then trypsinized and seeded onto the receiver cells at 1:200 ratios. The donor cells were allowed to attach to the receiver cell monolayer and form GJICs at $37^{\circ} \mathrm{C}$ for $4 \mathrm{~h}$, before being examined under a fluorescence microscope (Axio Imager A2; Carl Zeiss AG, Oberkochen, Germany). The receiver cells, which received Calcein from donor cells and should emit green fluorescence, were counted visually, and the images were captured. The average number of receiver cells containing Calcein per donor cell was then measured and calculated to deduce the function of GJIC.

2.9. Immunofluorescence Analysis. For immunofluorescence imaging, C4-2 cells were cultured in confocal Petri dishes with tangeretin $(0,3,10$, and $30 \mu \mathrm{m})$ for $24 \mathrm{~h}$. After three times of PBS rinsing, cells were fixed with $4 \%$ paraformaldehyde for $30 \mathrm{~min}$. Before the cells were blocked using $2 \%$ bovine serum albumin (BSA) for $30 \mathrm{~min}$ under room temperature, the cells were incubated with $0.1 \%$ Triton $\mathrm{X}-100$ for $20 \mathrm{~min}$. Subsequently, Cx26 primary antibodies (1:200; Bioss\#bs-1715R, China) were applied and incubated overnight in $4^{\circ} \mathrm{C}$. After rinsing with PBS, the cells were incubated with Alexa Fluor ${ }^{\circledR}$ 647-conjugated secondary antibodies (1:200; Abcam\#ab150075, Cambridge, UK) for $1 \mathrm{~h}$ under room temperature in a dark hood. Phalloidin $(5 \mu \mathrm{g} / \mathrm{ml})$ and DAPI $(1.43 \mu \mathrm{M}$; Abcam\#ab1176753, Cambridge, UK) were applied sequentially for actin and nuclear staining, respectively. After another round of PBS rinsing, fluorescent images of the cells were captured under a confocal microscope (LSM880; Carl Zeiss AG, Oberkochen, Germany).

2.10. Statistical Analysis. All experiments were repeated at least three times. SPSS 16.0 software (SPSS, Inc., Chicago, USA) was used for the statistical analysis of experimental data. An unpaired $t$-test was used to compare two groups. 
For two or more groups, one-way ANOVA was used to analyze the data. $P<0.05$ was considered to indicate a significant difference, where “*” represents a significant difference compared with the corresponding group. The histogram in the graph is expressed in the form of the mean \pm standard error.

\section{Results}

3.1. Network Pharmacology Study Analysis on the Potential Effects of Orange Peel on PCa. Network pharmacology approaches are increasingly being optimized and subsequently applied for exploring novel therapeutic strategies and repurposing previously approved drugs. The present study used this network pharmacology approach to analyze the mechanism underlying the effects of orange peel (citrus) for the potential treatment of PCa. A total of 63 compounds were identified to be associated with citrus from the TCMSP and SymMap databases in addition to the reported articles [28]. Following absorption, distribution, metabolism, excretion, and toxicity (ADME/T) calculations, 12 compounds were identified to be active and potentially effective compounds (Table 1). PCa-associated genes were then obtained from the OMIM and Genecards databases. The Venn diagram revealed the comparison and visualization of genes targeted by orange peel and genes associated with PCa (Figure 1(a)). There are 132 common targets of orange peel and $\mathrm{PCa}$, which are predicted to be key nodes through which citrus exerts its pharmacological effects. To explore these proteins, a PPI network between the orange peel and PCa was constructed and analyzed using STRING with a confidence score of $>0.9$ (Figure 1(b)). Based on these findings, a citrus-compoundtarget network was validated (Figure $1(\mathrm{c})$ ). GO analysis was then performed using the web-based DAVID tool, where 561 GO terms were assigned, which included 409 biological processes, 51 cellular components, and 101 molecular function terms $(P<0.05)$. Typically, enriched GO terms are shown in Figure 1(d). The molecular function categories were found to be particularly enriched in "protein binding" (107 differentially expressed genes (DEGs); $81.1 \%$ ). The highest percentages of GO terms under the cellular component and biological processes were "nucleus" (71 DEGs; 53.8\%) and "negative regulation of apoptotic process" (35 DEGs; 16.5\%). To identify the signaling pathways involved, KEGG databases were mapped, which found 96 KEGG pathways ( $Q$ value $<0.01$ ) to be significantly enriched (Figure $1(\mathrm{e})$ ). In particular, the DEGs were found to be highly clustered in a number of signaling pathways, including "pathways in cancer," "PI3K-AKT signaling pathway," and "Ras signaling pathways."

\subsection{Tangeretin Can Significantly Reduce Cell Viability and} Inhibit Colony Formation of CRPC Cells. In total, four compounds were chosen from the list of active components found in the orange peel to treat CRPC cells at the same concentrations $(10 \mu \mathrm{M})$. Among them, tangeretin was found to be the most effective in inhibiting the cell viability of C4-2 cells in the presence of 10\% FBS (Figure 2(a)). Therefore,
TABle 1: The 12 active compounds in citrus.

\begin{tabular}{lcccc}
\hline No. & Compound & OB (\%) & DL & $\mathrm{CaCo}_{2}$ \\
\hline C1 & Ledene & 51.84 & 0.1 & 1.86 \\
C2 & Sitosterol & 36.91 & 0.75 & 1.32 \\
C3 & Naringenin & 59.29 & 0.21 & 0.28 \\
C4 & Hesperetin & 47.74 & 0.27 & 0.28 \\
C5 & DIBP & 49.63 & 0.13 & 0.85 \\
C6 & Hepta-3 & 23.91 & 0.58 & 1.05 \\
C7 & Tangeretin & 21.38 & 0.43 & 1.23 \\
C8 & Citromitin & 86.9 & 0.51 & 0.88 \\
C9 & Nobiletin & 61.67 & 0.52 & 1.05 \\
C10 & Hesperidin & 13.33 & 0.67 & -2.03 \\
C11 & Neohesperidin & 11.57 & 0.69 & -2.05 \\
C12 & Hesperetin-5-glucoside & 21.82 & 0.83 & -1.42 \\
\hline
\end{tabular}

the effects of tangeretin on CRPC cells were focused upon. The effects of tangeretin on the viability of C4-2 and Du145 cells in serum-free conditions were then assessed using MTS assays. The results revealed that tangeretin can significantly inhibit the viability of Du145 (Figure 2(b)) and C4-2 cells (Figure 2(c)). In addition, tangeretin could significantly inhibit colony formation by A-375 (Figure 2(d)) and Du145 cells (Figure 2(e)).

\subsection{Tangeretin Can Synergistically Inhibit the Viability of} CRPC Cells alongside Cisplatin or Sorafenib in Vitro. Sorafenib and cisplatin are drugs that are typically applied for treating certain types of malignancies $[29,30]$. Some drugs can mediate the bystander effect following treatment with cisplatin or other anti-tumor drugs [31-33]. By the way, angiogenesis has a very important role in prostate tumors, and there are several studies showed the correlation [34, 35]. Sorafenib, a tyrosine kinase inhibitor, may exert its antiangiogenic effect [36]. Therefore, the combined effects of tangeretin and sorafenib or cisplatin in CRPC cells were next tested. Tangeretin was found to be able to synergistically inhibit the viability of C4-2 cells (Figure 3(a)) when combined with sorafenib in serum-free conditions. Tangeretin could also synergistically inhibit the viability of C4-2 cells (Figure 3(b)) and Du145 cells (Figure 3(c)) when combined with cisplatin under serum deprivation conditions. In addition, tangeretin synergistically inhibited colony formation by C4-2 cells (Figure 3(d)) when treated alongside sorafenib. As a result, it could be concluded that tangeretin can enhance the inhibitory effects of sorafenib and cisplatin on CRPC cell proliferation.

3.4. Tangeretin Can Synergistically Inhibit the Migration of CRPC Cells with Sorafenib In Vitro. Wound-healing assays were subsequently performed to explore the effects of tangeretin on the migration of CRPC cells. The results showed that tangeretin can significantly inhibit the migration of C4-2 and Du145 cells (Figures 3(e) and 3(f)) whilst also synergistically enhancing the inhibitory effects of sorafenib on Du145 cell migration. 

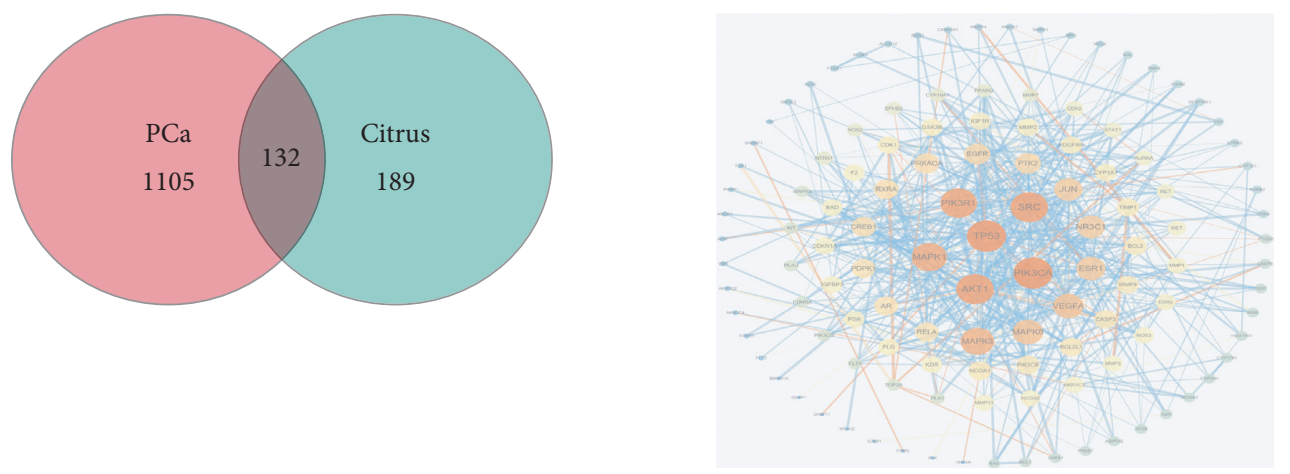

(a)

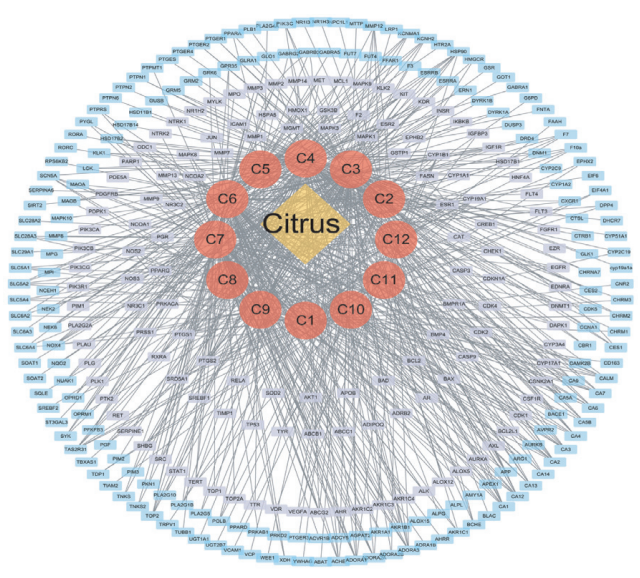

(b)
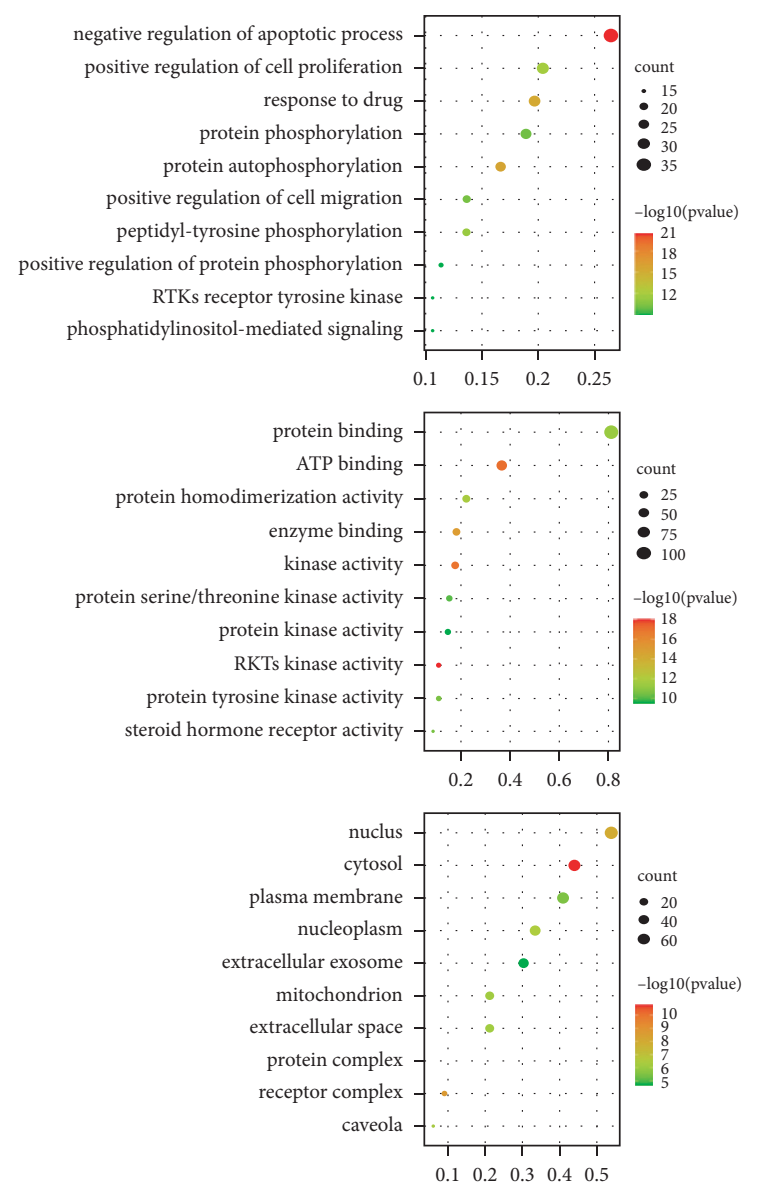

(c)

(d)

Figure 1: Continued. 


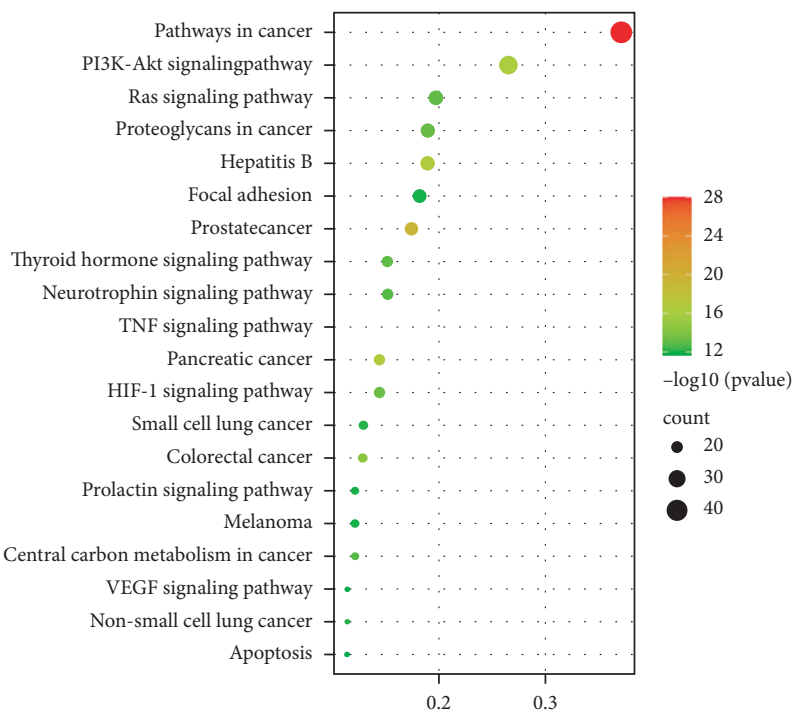

(e)

FIGURE 1: Network pharmacology analysis of the ingredients of orange peel and correlated targets of PCa. (a) Venn diagram showing the visualization of overlapped genes between those targeted by orange peel and those associated with PCa. (b) Protein-protein interaction network of orange peel and their associated targets in PCa. Higher degree values are represented by larger node sizes and brighter colors, whereas higher combined scores are represented by thicker edges and darker colors. Confidence score $>0.9$. (c) Orange peel-compoundtarget network. Yellow rhombi, red circles, blue rectangles, and purple rectangles represent orange peel; 12 active compounds were found within the orange peel; and the target genes were targeted by orange peel and overlapped genetic targets between PCa and orange peel, respectively. (d) Scatter plot of the top 10 gene ontology terms in the following three main categories: biological process, cellular component, and molecular function. (e) Scatter plot of the top 20 Kyoto Encyclopedia of Genes and Genomes pathways. The color and size of the dots represent the range of the $Q$ values, and the number of differentially expressed genes were mapped to the indicated pathways. PCa, prostate cancer.

3.5. Tangeretin Inhibits $A R$, Stat $3, A K T$, and $p A K T$ Expression in C4-2 Cells and Stat 3 and AKT Expression in Du145 Cells. A series of experiments were then performed to verify the potential effects of tangeretin on the predicted targets according to network pharmacology analysis. It was found that tangeretin could reduce Stat 3 and AKT expression in Du145 cells (Figure 4(a)) in addition to inhibiting AR, Stat3, AKT, and pAKT (Ser473) levels in C4-2 cells (Figure 4(b)). As a tyrosine kinase inhibitor, sorafenib can inhibit the PI3K/AKT signaling pathway $[37,38]$. Considering that AR plays a key role in prostate cancer, the possibility that tangeretin can synergize with sorafenib to reduce the expression of AR was assessed. It was revealed that tangeretin could synergize with sorafenib to inhibit AR and AKT expression in AR-positive C4-2 cells (Figure 4(c)).

3.6. Molecular Docking Analysis of Tangeretin AR and Cx26. To examine the molecular characteristics of tangeretin, the structures were obtained from PubChem (https://pubchem. ncbi.nlm.nih.gov/;Figure 5(a)). The crystal structures of AR (PDB ID: 5VO4) and Cx26 (PDB ID: 5ER7) were obtained from the PDB database (https://www.rcsb.org/); both structures were complexed with small molecules. The Autodock Vina was used to verify the aforementioned targets after uploading the three-dimensional structure of tangeretin. Through molecular docking, the interaction between tangeretin and the crystal structure of AR complexes (Figure 5(b)) was verified, yielding a docking score of -3.0 . In addition, interaction between tangeretin and the crystal structure of Cx26 complexes (PDB ID: 5ER7), a gap junction protein that can mediate the "bystander effect" by transmitting "death signals" to adjacent cells to enhance the killing effect of chemotherapy [39], was verified, which yielded a docking score of -6.3 (Figure $5(\mathrm{c})$ )). These results suggest that tangeretin can directly interact with $A R$ and Cx26 through hydrogen bonding.

3.7. Tangeretin Increases Cx26 Expression and GJIC. To verify the aforementioned molecular docking results, Cx26 expression was measured by western blotting, where the results revealed that tangeretin significantly increased Cx26 expression in both C4-2 and Du145 cells (Figures 6(a) and 6(b)). In addition, Cx26 expression and distribution were evaluated by immunofluorescence staining. The result showed that tangeretin treatment significantly increased the expression of Cx26 in C42 cells compared with that in untreated control cells (Figure 6(c)). The reduction or disappearance of GJs has been frequently associated with the development and growth of tumors [4]. Restoring GJ function in tumor cells can inhibit the proliferation of tumor cells by reprogramming the regulatory mechanism [40]. Therefore, GJIC function was next detected using parachute assay, which found that tangeretin treatment enhanced 


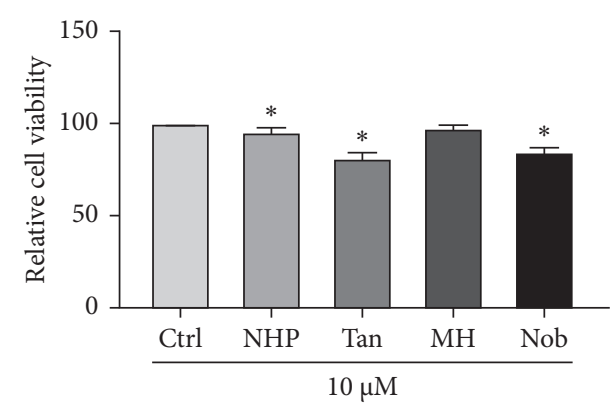

(a)

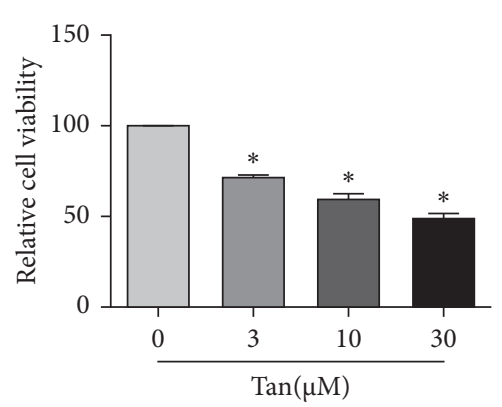

(b)

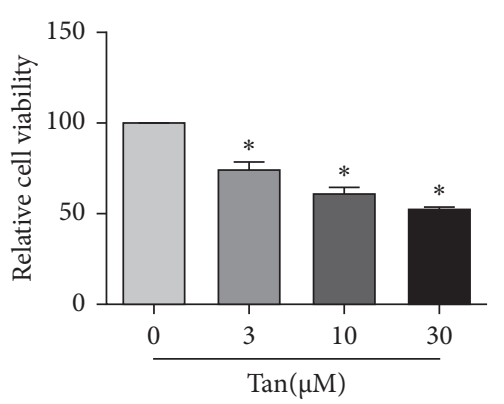

(c)

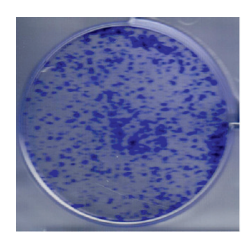

Control

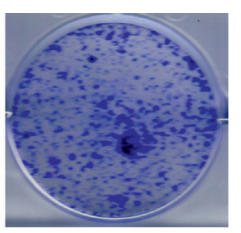

$\operatorname{Tan} 3 \mu \mathrm{M}$

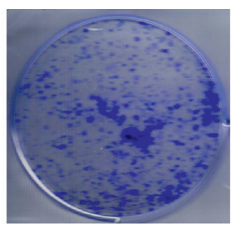

Tan $10 \mu \mathrm{M}$

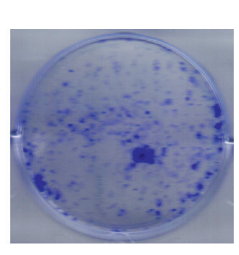

$\operatorname{Tan} 30 \mu \mathrm{M}$

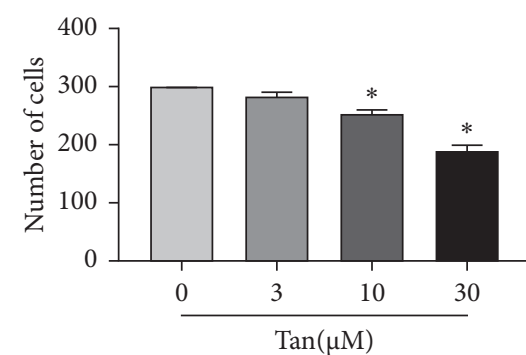

(d)

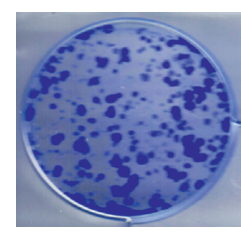

Control

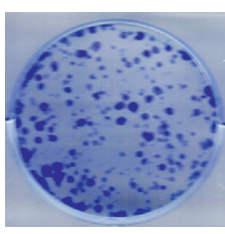

Tan $3 \mu \mathrm{M}$

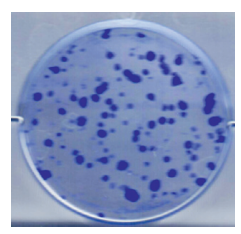

Tan $10 \mu \mathrm{M}$

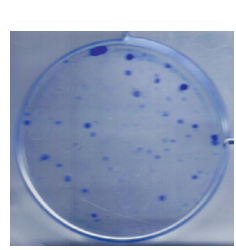

Tan $30 \mu \mathrm{M}$

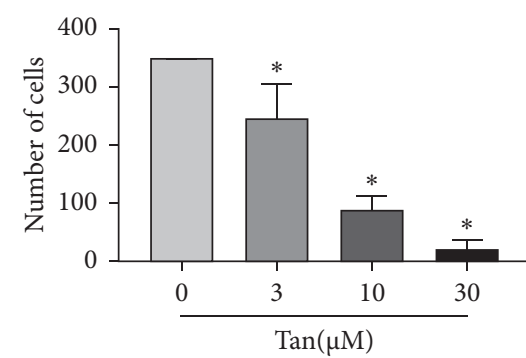

(e)

FIgURE 2: Tangeretin could significantly inhibit CRPC cell viability and colony formation. (a) Following the screening of four active compounds contained within orange peel by using them to treat CRPC cells at $10 \mu \mathrm{M}$ in $10 \%$ FBS, tangeretin was found to be the most effective in reducing C4-2 cell viability. (b) The effect of tangeretin on the viability of Du145 cells in serum-free conditions was detected using an MTS assay. (c) Tangeretin can significantly reduce the viability of C4-2 cells in serum-free conditions. Tangeretin can significantly inhibit colony formation by (d) A-375 and (e) Du145 cells. ${ }^{*} P<0.05$ compared with the control or the 0 group. CRPC, castration-resistant prostate cancer; Tan, tangeretin; NHP, neohesperidin; MH, methyl-hesperidin; and Nob, 5-Nobiletin.

GJIC function among C4-2 cells (Figure 6(d)) and Du145 cells (Figure 6(e)). Taken together, tangeretin could significantly increase Cx26 expression whilst enhancing GJIC in CRPC cells.

\subsection{Cx26 Overexpression Inhibits $A K T$ and $A R$ Expression.} To decipher the associations and interactions among the aforementioned molecules, Cx26-overexpressing cell lines of C4-2 were constructed. The results showed Cx26 overexpression could inhibit the expression of AKT and AR (Figures 6(f) and 6(i)) whilst also reducing the viability of C4-2 cells (Figure 6(g)).

According to the target genes of tangeretin, a PPI network was constructed using the STRING tool (Figure 6(h)). From the PPI network, although the predicted direct relationships among the molecules aforementioned could be observed, Cx26 was not found to be one of the core molecules according to these predictions based on previous data.
However, this observation provided a novel perspective on the role of Cx26 in AKT and AR expression.

Taken together, data from the present study revealed that tangeretin can inhibit the proliferation of CRPC cells by upregulating Cx26 expression, which in turn inhibited AKT and AR expression to synergistically enhance the sensitivity of CRPC cells to cisplatin and sorafenib.

\section{Discussion}

In the present study, the key components and targets of orange peel, a traditional Chinese medicine, were first analyzed using a network pharmacology method. This screened out 12 predicted compounds contained within orange peel from the TCMSP database, where their corresponding targets were subsequently predicted. By integrating these with the PCa-associated disease genes, 132 overlapped genes and drug targets were found. To confirm the relationship of these compounds further with $\mathrm{PCa}$, a corresponding 


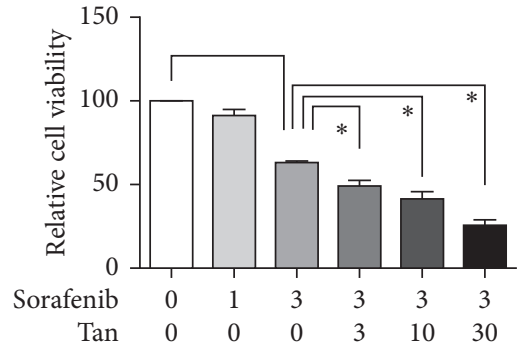

(a)

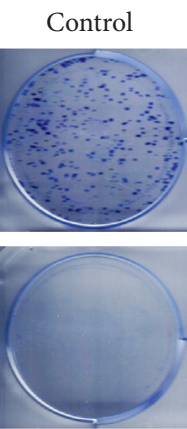

Tan $30 \mu \mathrm{M}$

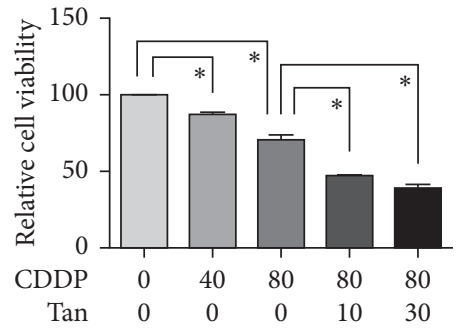

(b)

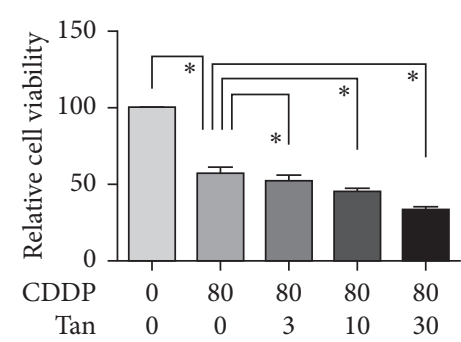

(c)

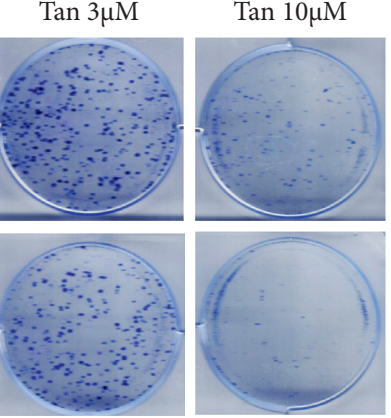

Tan $0 \mu \mathrm{M}$

Tan $10 \mu \mathrm{M}$

Sorafenib $1 \mu \mathrm{M}$

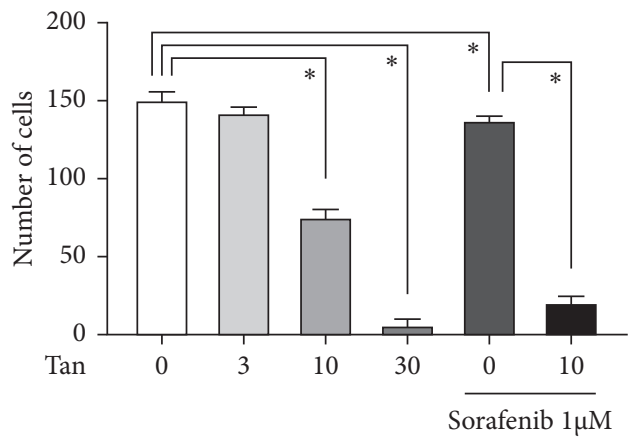

(d)

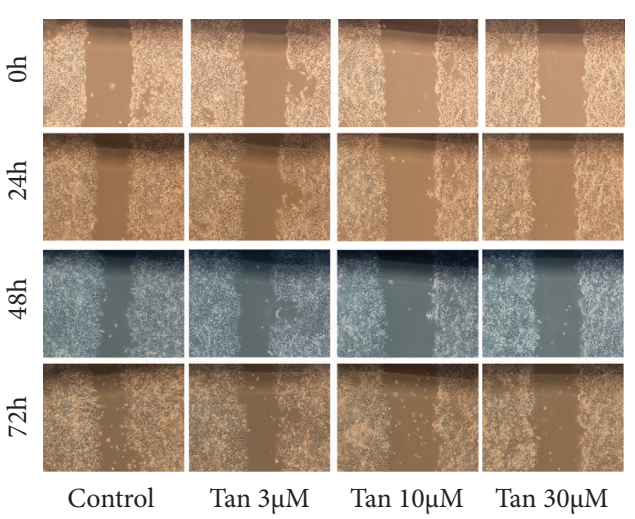

(e)

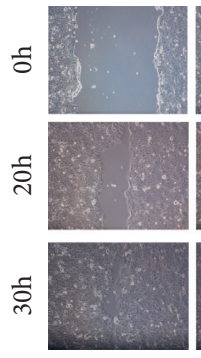

Control

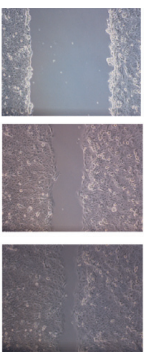

Tan $3 \mu \mathrm{M}$

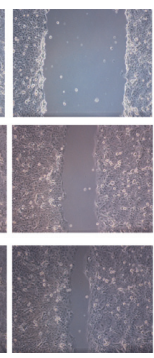

Tan $10 \mu \mathrm{M}$

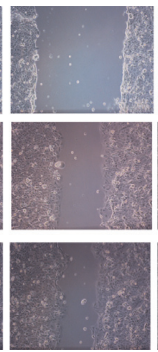

Tan $30 \mu \mathrm{M}$
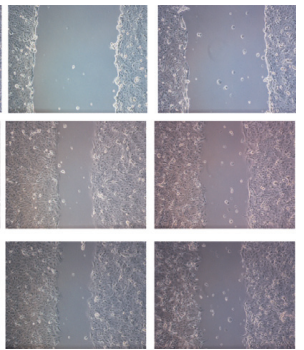

$\operatorname{Tan} 30 \mu \mathrm{M}$

Sorafenib $3 \mu \mathrm{M}$

(f)

FIGURE 3: Tangeretin could synergistically inhibit the proliferation and migration of castration-resistant prostate cancer cells when combined with cisplatin or sorafenib under serum deprivation conditions. (a) Tangeretin can synergistically reduce the viability of C4-2 cells when treated alongside sorafenib in serum-free conditions. (b) Tangeretin can also synergistically reduce the viability of C4-2 cells when combined with cisplatin under serum deprivation conditions. (c) Tangeretin can synergistically reduce the cell viability of Du145 cells when combined with cisplatin in serum-free conditions. (d) Tangeretin can significantly inhibit colony formation by C4-2 cells synergy when combined with sorafenib. (e) Tangeretin can significantly inhibit the migration of C4-2 cells $(\times 200)$. (f) Tangeretin can significantly inhibit the migration of Du145 cells, whilst also synergistically inhibiting their migration when combined with sorafenib under reduced serum conditions $(\times 200)$. ${ }^{*} P<0.05$ compared with the control or the 0 group. Tan, tangeretin and CDDP, cisplatin.

compound-target-signaling pathway network was also constructed. It was found that tangeretin, an active ingredient from orange peel, could significantly inhibit CRPC cell (C4-2 and Du145) proliferation and migration, whilst synergistically enhancing their sensitivity to sorafenib and cisplatin. Tangeretin also significantly reduced AR and AKT expression in C4-2 cells in addition to reducing Stat3 in androgen-insensitive Du145 cells.

Additionally, tangeretin increased the expression of both Cx26 and enhanced GJ function in CRPC cells, which may mediate the bystander effect following treatment with cisplatin or other anti-tumor drugs [31-33]. Therefore, Cx26overexpressing cell lines were then constructed, where it was found that Cx26 overexpression inhibited AR and AKT signaling. Therefore, $\mathrm{Cx} 26$ may serve to be a novel target that can be manipulated to hinder the development and progression of $\mathrm{PCa}$ and AKT/AR signaling (Supplementary Figure 1).

Almost all mammalian cells are connected through GJs. Small molecules, such as calcium ions, $\mathrm{IP}_{3}$, and cAMP, can be 

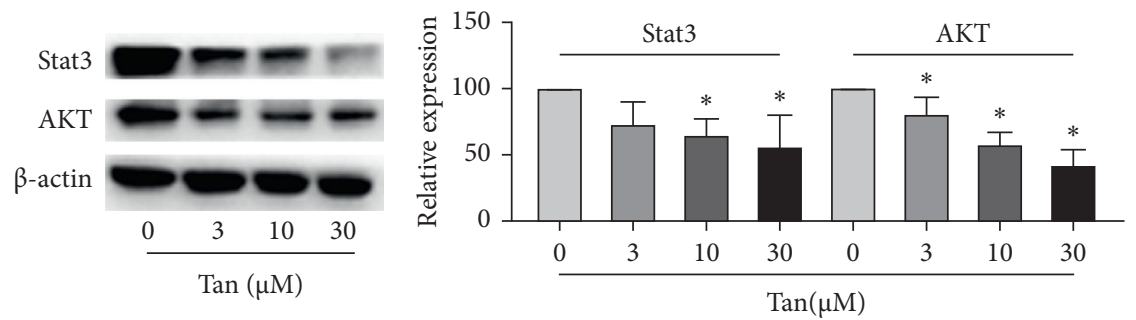

(a)
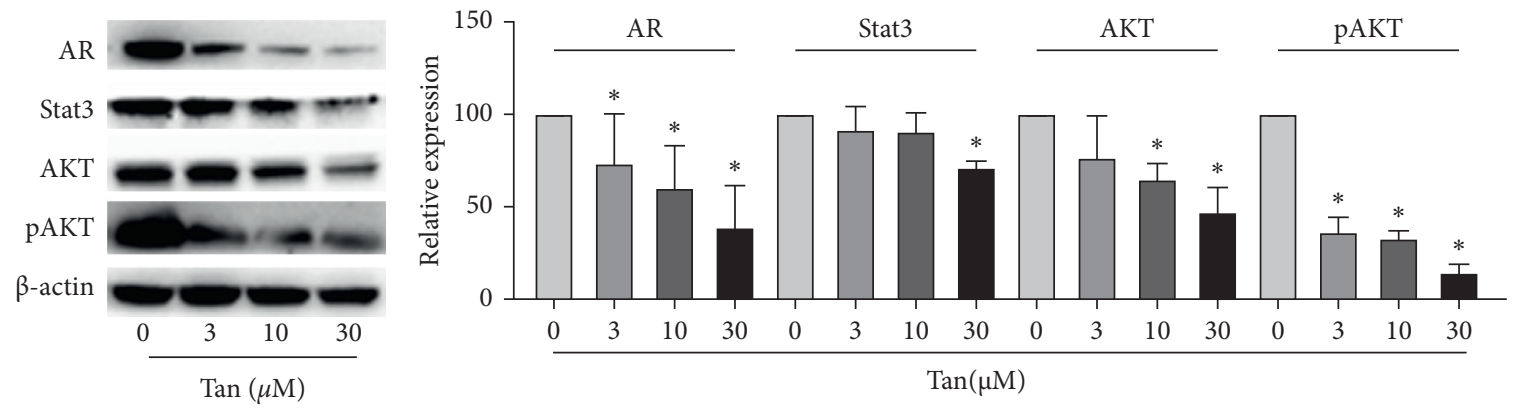

(b)
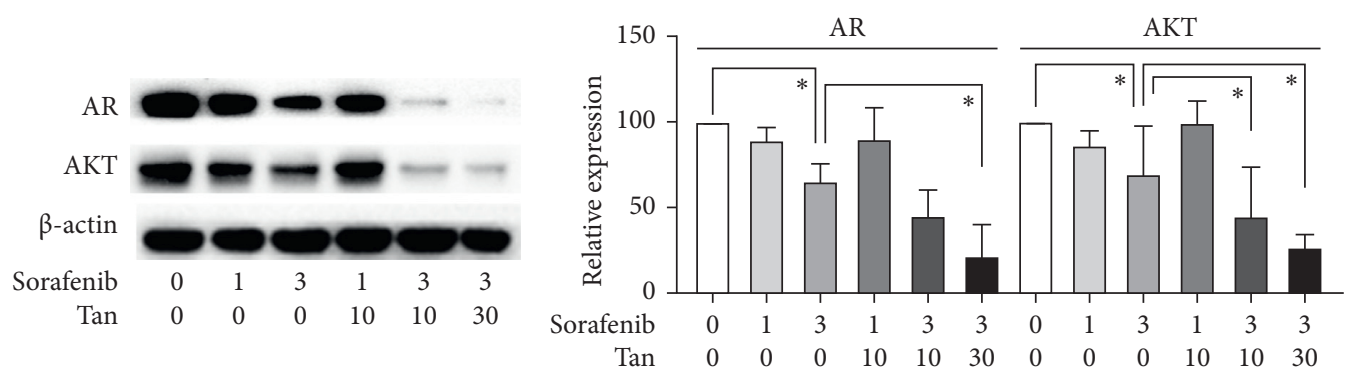

(c)

FIgURE 4: Tangeretin could inhibit AR, Stat3, and AKT expression in castration-resistant prostate cancer cells. (a) Tangeretin inhibits Stat3 and AKT expression in Du145 cells. (b) Tangeretin reduces AR, Stat3, AKT, and pAKT protein levels in C4-2 cells. (c) Tangeretin enhances the inhibitory effects of sorafenib on AR expression in C4-2 cells. ${ }^{*} P<0.05$ compared with the 0 group. Tan, trangeretin; AR, androgen receptor; Stat3, signal transducer and activator of transcription 3; AKT, protein kinase B; and p, phosphorylated.

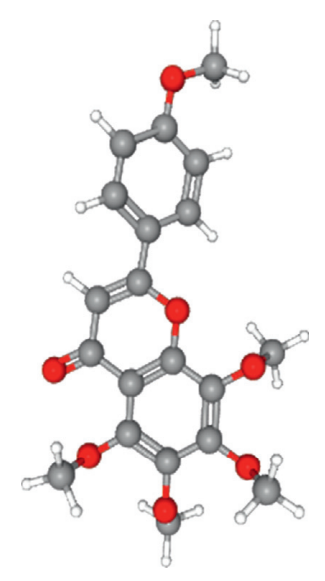

(a)

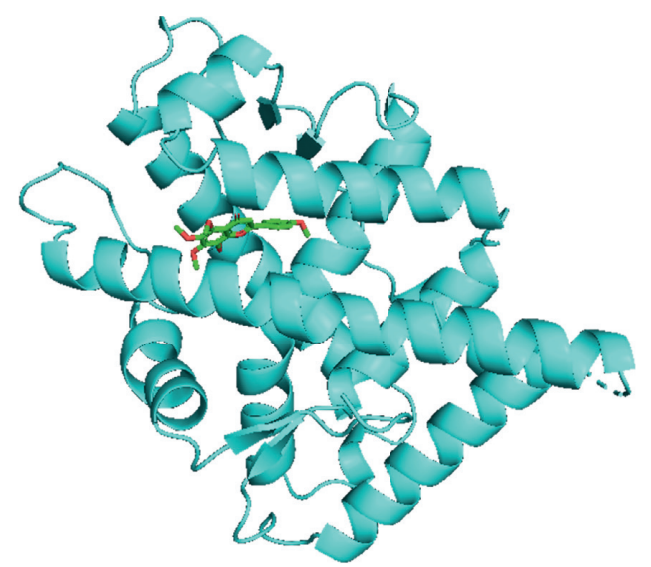

(b)

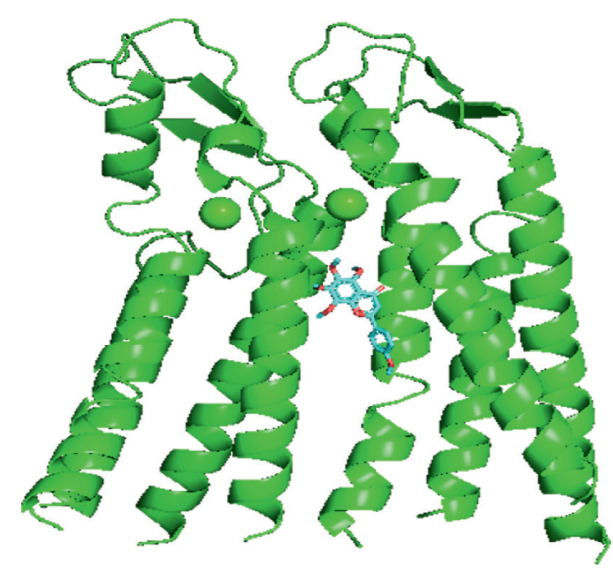

(c)

Figure 5: Molecular docking analysis of tangeretin, AR, and Cx26. (a) Molecular structure of tangeretin. (b) Molecular docking of tangeretin onto the crystal structure of AR complexes (PDB ID: 5VO4) with a docking score of -3.0. (c) Molecular docking of tangeretin onto the crystal structure of Cx26 complexes (PDB ID: 5ER7) with a docking score of -6.3 . The dotted green line represents hydrogen bonding. AR, androgen receptor and Cx26, connexin 26. 


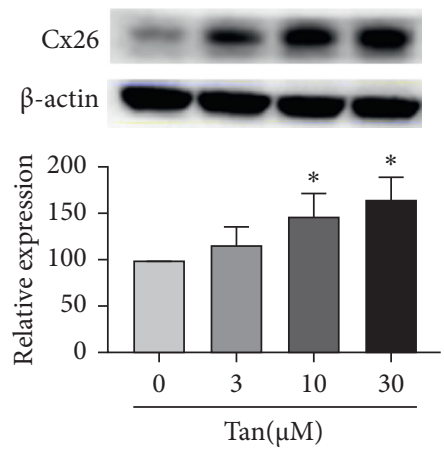

(a)

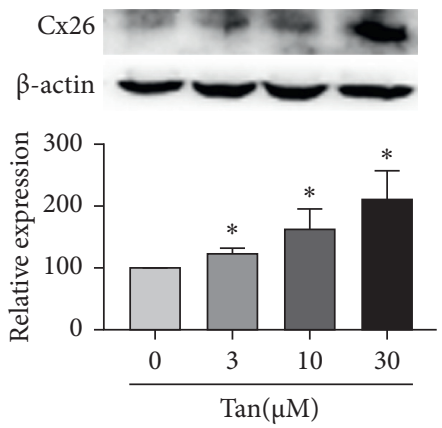

(b)

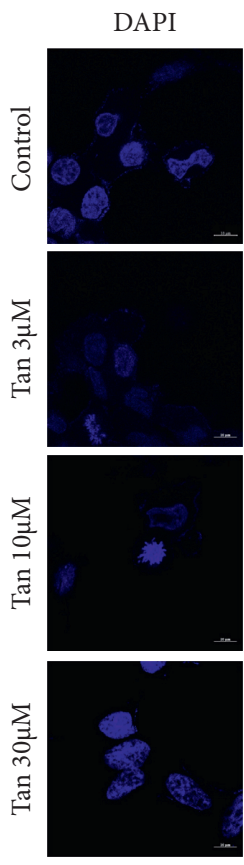

Phalloidin
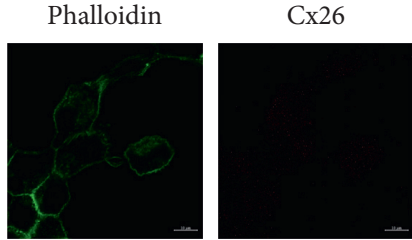

Merge
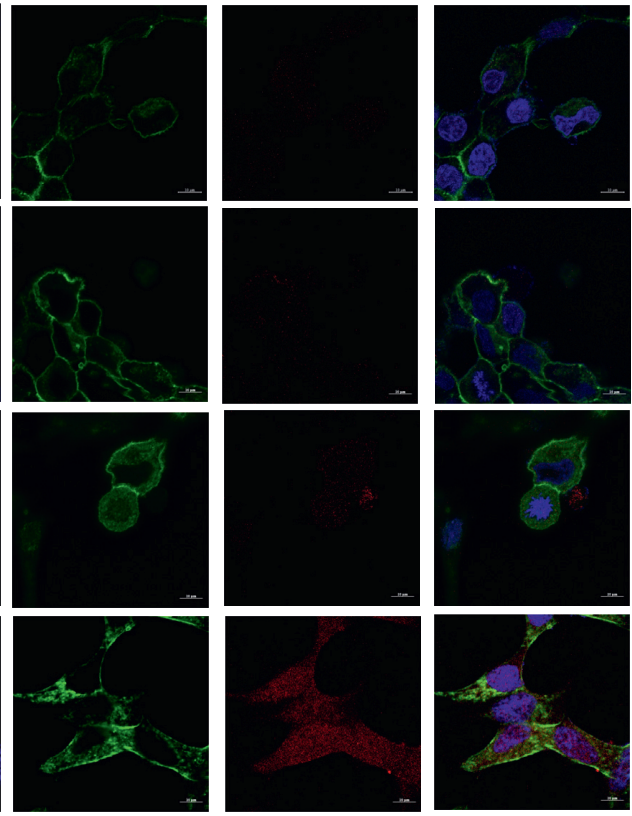

(c)

Figure 6: Continued. 

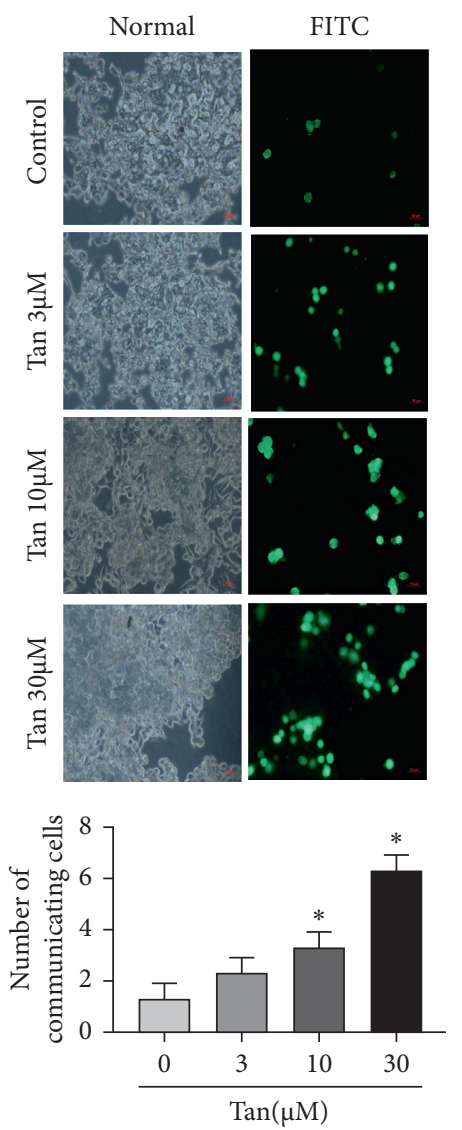

(d)
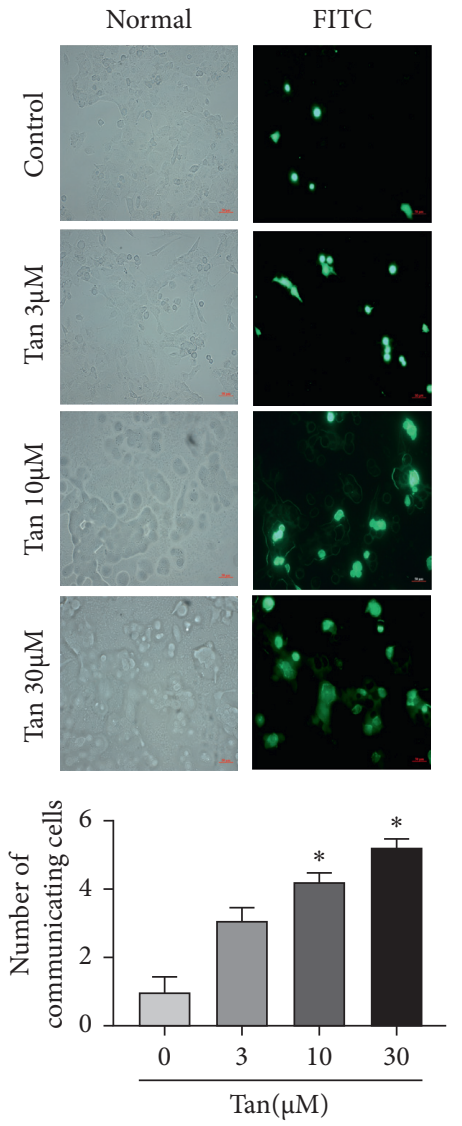

(e)

FIgURE 6: Continued. 


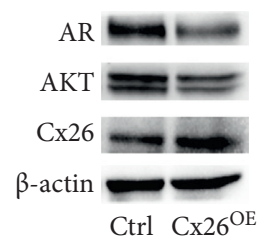

(f)

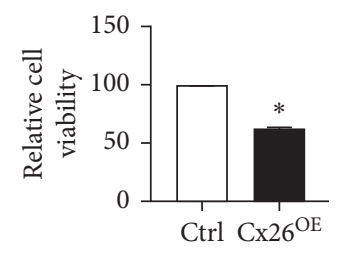

(g)

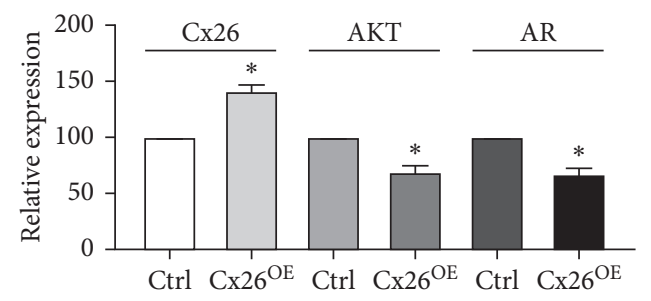

(h)

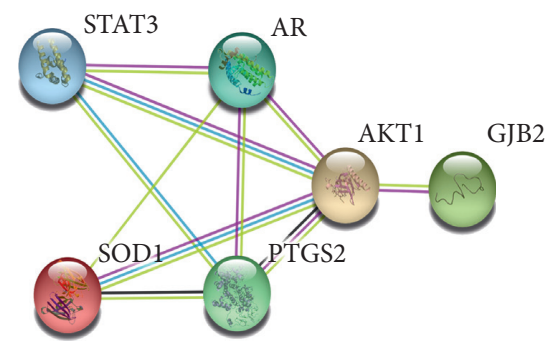

(i)

FIGURE 6: Tangeretin increased Cx26 expression and facilitated AKT and AR signaling to promote GJIC function in castration-resistant prostate cancer cells. (a) Tangeretin can significantly increase Cx26 expression in C4-2 cells. (b) Tangeretin can significantly increase Cx26 expression in Du145 cells. (c) Immunofluorescence analysis confirmed that tangeretin can increase Cx26 expression (10 × 100). (d) Functional GJIC was detected using parachute assay in C4-2 cells ( $\times 200)$. (e) Functional GJIC was detected using parachute assay in Du145 cells $(\times 200)$. (f) Cx26 overexpression can significantly inhibit the expression of AKT and AR in C4-2 cells. (g) Cx26 overexpression can reduce the viability of C4-2 cells as detected by MTS assay $72 \mathrm{~h}$ after transfection. (h) Representative western blotting images and quantification of Cx26, AKT, and AR protein expression in C4-2 cells and Cx26-overexpressing C4-2 cells. (i) Protein-protein interaction network of tangeretin and target genes following search tool for the retrieval of interacting genes/proteins analysis. Confidence score $>0.4$. ${ }^{*} P<0.05$ compared with the control or the 0 group. Tan, tangeretin; Cx26, connexin 26; AKT, protein kinase B; AR, androgen receptor; and GJIC, gap junctional intercellular communication.

transmitted among cells through GJs, such that cells can share metabolites to regulate the signal transmission status of each other. GJ serves an important role in the occurrence, growth, invasion, and metastasis of tumors in addition to the response to therapy. During tumor chemotherapy or radiotherapy, cells can transmit "death signals" to adjacent cells by GJs to promote apoptosis, thereby enhancing the killing effect, in a phenomenon known as the "bystander effect" [41, 42]. Through molecular docking analysis, it was revealed that Cx26, a gap junction protein, may be a target of tangeretin. Cx26 genes are located on chromosome 13 [43]. It is mainly expressed in epithelial cells and serves an important role in maintaining the normal flow of materials and signals among cells in the urothelial tract. In particular, there are large quantities of glandular and basal epithelial tissues in the prostate.
Previous studies have shown that the expression levels of Cx26 were negatively correlated with the malignancy severity of transitional cell carcinoma [44]. Transfection with the $\mathrm{Cx} 26$ adenovirus was found to inhibit the proliferation of a variety of PCa cell lines in vitro, such as PC-3, LNCAP, and DU-145, by inducing cell cycle arrest at the $G_{2}$ or $M$ phases to promote apoptosis [45]. In addition, Cx26 can reduce the invasion and metastasis of tumor cells by inhibiting adhesion kinase adhesion [46]. The present study also revealed that tangeretin can inhibit the proliferation of CRPC cells by inducing Cx26-mediated inhibition of AKT and AR signaling whilst facilitating the formation of GJIC. In addition, the GJIC formed by Cx26 could promote the "bystander effect" following treatment with sorafenib or cisplatin. Therefore, both junctional and nonjunctional inhibitory 
effects mediated by Cx26 were presented. Since Cx26 expression could inhibit the proliferation of CRPC cells, the specific role of $\mathrm{Cx} 26$ in the development of $\mathrm{PCa}$ is anticipated to be unraveled by future investigations.

By regulating nutrient metabolism, cell proliferation, survival, migration, and angiogenesis, the PI3K/AKT signaling pathway was found to be activated in a wide variety of cancer types [47]. AKT/mTOR [48] and AR signaling pathways [49] are aberrantly activated in prostate cancer, where the mechanism between the PI3K/AKT/mTOR pathway and several key oncogenic signaling cascades, such as AR, mitogen-activated protein kinase (MAPK), and Wnt signaling cascades [50], underlying PCa growth and drug resistance has been previously discussed [51]. Tangeretin has been reported to inhibit the proliferation of PCa cells by targeting the PI3K/AKT/mTOR signaling pathway [24]. The present study therefore selected two CRPC cell lines, where the results not only confirmed AKT inhibition by tangeretin but also discovered that tangeretin can inhibit AR expression, which serves a key role in the development of PCa and CRPC. However, tangeretin has been found to have inhibitory effects on a variety of tumors $[12,52]$, it remains to be further explored whether tangeretin can affect the progression of other tumors by inhibiting AR expression and whether it can also exert inhibitory effects on other tumors through Cx26/AKT/AR signaling pathway.

AR signaling is a hub that laid the foundation for a number of signaling mechanisms in CRPC, where it serves a central role in mediating the process of androgen-dependent progression to CRPC. The mechanism underlying AR triggering in CRPC mainly includes AR point mutations, AR overexpression, and AR coactivators, all of which promote the abnormal activation of AR [53]. Therefore, AR is also a therapeutic target for CRPC. Apart from the AR signaling pathway, receptor tyrosine kinases and AKT1 have also been previously associated with PCa [54]. AR mutations frequently occur in the ligand-binding domain (LBD) of $A R$, where the most common mutation is the T877 A mutation [55]. However, the role of tangeretin on AR full length (AR-FL) and AR-splice variants (AR-Vs) require further investigation.

In the present study, tangeretin was selected to be the key research target from the orange peel. Another citrus compound naringenin was found to induce apoptotic cell death in PCa cells via the PI3K/AKT and MAPK signaling pathways [56], which is consistent with the present study. At present, the protective effects of citrus on PCa have been reported by epidemiological analysis, but the corresponding effects of citrus and tangeretin on CRPC in adjuvant diet regimen require further verification by additional clinical evidence. As one of the active ingredients of orange peel that is readily available and more economically viable than abiraterone, tangeretin can be used as a dietary supplement in daily life, which may serve as a synergistic treatment strategy whilst reducing toxic side effects and preventing drug resistance in patients.

However, more experimental and clinical evidence were needed to determine the relationship between tangerine peel and citrus and prostate cancer. In addition, due to the influence of water solubility, the antiprostate cancer activity and bioavailability of citrine still need to be further optimized. The development of AR-targeting drugs based on tangeretin and orange peel may reduce the treatment burden of patients with PCa to some extent. The structural modification and exploitation of using tangeretin to target $\mathrm{AR}$ may provide a novel direction for the development of dietary compounds for PCa treatment.

\section{Conclusion}

The present study revealed that orange peel and its active compound tangeretin can inhibit the proliferation, migration, and colony formation of CRPC cells. In addition, strong indications were provided that tangeretin can inhibit AR expression, which serves a key role in the development of PCa. By inducing the expression of $\mathrm{Cx} 26$, tangeretin can inhibit AKT and AR expression whilst facilitating the formation of GJIC. These results may have an impact on the therapy of CRPC and for the food and medicine industry with regard to using orange peel.

\section{Abbreviations}

ADPC: Androgen-dependent prostate cancer

Akt: $\quad$ Protein kinase B

AR: Androgen receptor

CRPC: Castration-resistant prostate cancer

Cx26: Connexin 26

GJIC: Gap junction intercellular communication

Tan: Tangeretin.

\section{Data Availability}

The data used to support the findings of this study are included within the article.

\section{Conflicts of Interest}

The authors declare that they have no conflicts of interest.

\section{Authors' Contributions}

Ningfang Zhang, Wenqi Wu, and Yapeng Huang contributed equally to this work.

\section{Acknowledgments}

This work was supported by research grants from the National Natural Science Foundation of China (no. 81803576).

\section{Supplementary Materials}

Supplementary Figure 1. Tangeretin significantly inhibits AR and AKT expression in CRPC cells. Intriguingly, tangeretin increases both the expression of the connexin26 (Cx26) and the function of gap junction, which may mediate the bystander effect of antitumor drugs. (Supplementary Materials) 


\section{References}

[1] D. Senapati, S. Kumari, and H. V. Heemers, "Androgen receptor co-regulation in prostate cancer," Asian Journal of Urology, vol. 7, no. 3, pp. 219-232, 2020.

[2] D. T. Miyamoto, Y. Zheng, B. S. Wittner et al., "RNA-Seq of single prostate CTCs implicates noncanonical Wnt signaling in antiandrogen resistance," Science, vol. 349, no. 6254, pp. 1351-1356, 2015.

[3] S. Wang, C. Gilbreath, R. K. Kollipara et al., "Mithramycin suppresses DNA damage repair via targeting androgen receptor in prostate cancer," Cancer Letters, vol. 488, pp. 40-49, 2020.

[4] D. Banerjee, "Connexin's connection in breast cancer growth and progression," International Journal of Cell Biology, vol. 2016, Article ID 9025905, 11 pages, 2016.

[5] J.-I. Wu and L.-H. Wang, "Emerging roles of gap junction proteins connexins in cancer metastasis, chemoresistance and clinical application," Journal of Biomedical Science, vol. 26, no. 1, p. 8, 2019.

[6] Y. Zhang, L. Tao, L. Fan et al., "Different gap junctionpropagated effects on cisplatin transfer result in opposite responses to cisplatin in normal cells versus tumor cells," Scientific Reports, vol. 5, no. 1, Article ID 12563, 2015.

[7] M. Uzu, H. Sato, R. Yamada et al., "Effect of enhanced expression of connexin 43 on sunitinib-induced cytotoxicity in mesothelioma cells," Journal of Pharmacological Sciences, vol. 128, no. 1, pp. 17-26, 2015.

[8] Y. Kou, L. Ji, H. Wang et al., "Connexin 43 upregulation by dioscin inhibits melanoma progression via suppressing malignancy and inducing M1 polarization," International Journal of Cancer, vol. 141, no. 8, pp. 1690-1703, 2017.

[9] Y. Lai, L. Fan, Y. Zhao et al., "Cx32 suppresses extrinsic apoptosis in human cervical cancer cells via the NF- $\kappa \mathrm{B}$ signalling pathway," International Journal of Oncology, vol. 51, no. 4, pp. 1159-1168, 2017.

[10] C. J. Weng and G. C. Yen, "Flavonoids, a ubiquitous dietary phenolic subclass, exert extensive in vitro anti-invasive and in vivo anti-metastatic activities," Cancer \& Metastasis Reviews, vol. 31, no. 1-2, pp. 323-351, 2012.

[11] C. Chaumontet, C. Droumaguet, V. Bex, C. Heberden, I. Gaillard-Sanchez, and P. Martel, "Flavonoids (apigenin, tangeretin) counteract tumor promoter-induced inhibition of intercellular communication of rat liver epithelial cells," Cancer Letters, vol. 114, no. 1-2, pp. 207-210, 1997.

[12] W. Raza, S. Luqman, and A. Meena, "Prospects of tangeretin as a modulator of cancer targets/pathways," Pharmacological Research, vol. 161, Article ID 105202, 2020.

[13] M. K. Kang, S. I. Kim, S. Y. Oh, W. Na, and Y. H. Kang, "Tangeretin ameliorates glucose-induced podocyte injury through blocking epithelial to mesenchymal transition caused by oxidative stress and hypoxia," International Journal of Molecular Sciences, vol. 21, no. 22, 2020.

[14] M. Li, Y. Zhao, D. Qi, J. He, and D. Wang, "Tangeretin attenuates lipopolysaccharide-induced acute lung injury through notch signaling pathway via suppressing Th17 cell response in mice," Microbial Pathogenesis, vol. 138, Article ID 103826, 2020.

[15] E.-S. A. Arafa, Q. Zhu, B. M. Barakat et al., "Tangeretin sensitizes cisplatin-resistant human ovarian cancer cells through downregulation of phosphoinositide 3-kinase/Akt signaling pathway," Cancer Research, vol. 69, no. 23, pp. 8910-8917, 2009.
[16] H. H. Arab, W. R. Mohamed, B. M. Barakat, and E.-S. A. Arafa, “Tangeretin attenuates cisplatin-induced renal injury in rats: impact on the inflammatory cascade and oxidative perturbations," Chemico-Biological Interactions, vol. 258, pp. 205-213, 2016.

[17] A. Lakshmi and S. P. Subramanian, "Tangeretin ameliorates oxidative stress in the renal tissues of rats with experimental breast cancer induced by 7,12-dimethylbenz[a]anthracene," Toxicology Letters, vol. 229, no. 2, pp. 333-348, 2014.

[18] A. Geraci, V. Di Stefano, E. Di Martino, D. Schillaci, and R. Schicchi, "Essential oil components of orange peels and antimicrobial activity," Natural Product Research, vol. 31, no. 6, pp. 653-659, 2017.

[19] C. Yang, H. Chen, H. Chen, B. Zhong, X. Luo, and J. Chun, "Antioxidant and anticancer activities of essential oil from gannan navel orange peel,” Molecules, vol. 22, no. 8, 2017.

[20] K. Sak, H. Lust, M. Kase, M. Saar, and J. Jaal, "Suppression of taxanes cytotoxicity by citrus flavonoid hesperetin in PPC-1 human prostate cancer cells," Anticancer Research, vol. 38, no. 11, pp. 6209-6215, 2018.

[21] S. Conti, A. Vexler, L. Hagoel et al., "Modified citrus pectin as a potential sensitizer for radiotherapy in prostate cancer," Integrative Cancer Therapies, vol. 17, no. 4, pp. 1225-1234, 2018.

[22] A. Perez-Cornago, R. C. Travis, P. N. Appleby et al., "Fruit and vegetable intake and prostate cancer risk in the European Prospective Investigation into Cancer and Nutrition (EPIC)," International Journal of Cancer, vol. 141, no. 2, pp. 287-297, 2017.

[23] G.-J. Wei, Y.-H. Chao, Y.-C. Tung, T.-Y. Wu, and Z.-Y. Su, “A tangeretin derivative inhibits the growth of human prostate cancer LNCaP cells by epigenetically restoring p21 gene expression and inhibiting cancer stem-like cell proliferation," The AAPS Journal, vol. 21, no. 5, p. 86, 2019.

[24] W. B. Zhu, N. Xiao, and X. J. Liu, "Dietary flavonoid tangeretin induces reprogramming of epithelial to mesenchymal transition in prostate cancer cells by targeting the PI3K/Akt/ mTOR signaling pathway," Oncology Letters, vol. 15, no. 1, pp. 433-440, 2018.

[25] K. Yu, P. Zhang, and Z. G. Xie, "A network pharmacology study on the mechanisms of the herbal extract, christina loosestrife, for the treatment of nephrolithiasis," Medical Science Monitor: International Medical Journal of Experimental and Clinical Research, vol. 26, Article ID e919360, 2020.

[26] Y. Lai, X. Liang, F. Zhong et al., "Allicin attenuates calcium oxalate crystal deposition in the rat kidney by regulating gap junction function," Journal of Cellular Physiology, vol. 234, no. 6, pp. 9640-9651, 2019.

[27] Y. Zhao, Y. Lai, H. Ge et al., "Non-junctional Cx32 mediates anti-apoptotic and pro-tumor effects via epidermal growth factor receptor in human cervical cancer cells," Cell Death \& Disease, vol. 8, no. 5, Article ID e2773, 2017.

[28] Y. P. Song, H. F. Chen, and S. S. Tan, “A comparative study on content of hesperidin, Nobiletin, Tangeretin a synephrine in pericarpium citri reticulatae from different varieties," Lishizhen Medicine and Materia Research, vol. 28, no. 9, pp. 2061-2064, 2017.

[29] W. Tang, Z. Chen, W. Zhang et al., "The mechanisms of sorafenib resistance in hepatocellular carcinoma: theoretical basis and therapeutic aspects," Signal Transduction and Targeted Therapy, vol. 5, no. 1, p. 87, 2020.

[30] G. de Vries, X. Rosas-Plaza, M. A. T. M. van Vugt, J. A. Gietema, and S. de Jong, "Testicular cancer: determinants 
of cisplatin sensitivity and novel therapeutic opportunities," Cancer Treatment Reviews, vol. 88, Article ID 102054, 2020.

[31] S. Arora, J. R. Heyza, E. C. Chalfin, R. J. Ruch, and S. M. Patrick, "Gap junction intercellular communication positively regulates cisplatin toxicity by inducing DNA damage through bystander signaling," Cancers, vol. 10, no. 10, 2018.

[32] P. Samuel, L. A. Mulcahy, F. Furlong et al., "Cisplatin induces the release of extracellular vesicles from ovarian cancer cells that can induce invasiveness and drug resistance in bystander cells," Philosophical Transactions of the Royal Society of London-Series B: Biological Sciences, vol. 373, 1737 pages, 2018.

[33] I. J. Van Dillen, N. H. Mulder, and W. J. Sluiter, "Consequences of chemoresistance for the herpes simplex virus thymidine kinase/ganciclovir-induced bystander effect in a human small cell lung cancer cell line model," Anticancer Research, vol. 25, no. 1, pp. 255-261, 2005.

[34] N. Weidner, P. R. Carroll, J. Flax, W. Blumenfeld, and J. Folkman, "Tumor angiogenesis correlates with metastasis in invasive prostate carcinoma," American Journal of Pathology, vol. 143, no. 2, pp. 401-409, 1993.

[35] J. L. F. Duque, K. R. Loughlin, R. M. Adam, P. W. Kantoff, D. Zurakowski, and M. R. Freeman, "Plasma levels of vascular endothelial growth factor are increased in patients with metastatic prostate cancer," Urology, vol. 54, no. 3, pp. 523-527, 1999.

[36] M. Merino, A. Pinto, R. González, and E. Espinosa, "Antiangiogenic agents and endothelin antagonists in advanced castration resistant prostate cancer," European Journal of Cancer, vol. 47, no. 12, pp. 1846-1851, 2011.

[37] J. Zhang, J. Yu, R. Xie, W. Chen, and Y. Lv, "Combinatorial anticancer effects of curcumin and sorafenib towards thyroid cancer cells via PI3K/Akt and ERK pathways," Natural Product Research, vol. 30, no. 16, pp. 1858-1861, 2016.

[38] Z. Y. Zhang, S. M. Dong, Y. H. Liu et al., "Enhanced anticancer activity by the combination of vinpocetine and sorafenib via PI3K/AKT/GSK- $3 \beta$ signaling axis in hepatocellular carcinoma cells," Anti-Cancer Drugs, vol. 32, no. 7, pp. 727-733, 2021.

[39] L. Garcia-Rodríguez, S. Pérez-Torras, and M. Carrió, "Connexin-26 is a key factor mediating gemcitabine bystander effect," Molecular Cancer Therapeutics, vol. 10, no. 3, pp. 505-517, 2011.

[40] M. Artesi, J. Kroonen, M. Bredel et al., "Connexin 30 expression inhibits growth of human malignant gliomas but protects them against radiation therapy," Neuro-Oncology, vol. 17, no. 3, pp. 392-406, 2015.

[41] E. E. Mulkearns-Hubert, O. Reizes, and J. D. Lathia, "Connexins in cancer: jekyll or hyde?” Biomolecules, vol. 10, no. 12, 2020.

[42] C. X. Chen, K. J. Luo, and J. P. Yang, "Connexins and cAMP cross-talk in cancer progression and metastasis," Cancers, vol. 13, no. 1, 2020.

[43] G. I. Fishman, R. L. Eddy, T. B. Shows, L. Rosenthal, and L. A. Leinwand, "The human connexin gene family of gap junction proteins: distinct chromosomal locations but similar structures," Genomics, vol. 10, no. 1, pp. 250-256, 1991.

[44] J. Gee, M. Tanaka, and H. B. Grossman, "Connexin 26 is abnormally expressed in bladder cancer," The Journal of Urology, vol. 169, no. 3, pp. 1135-1137, 2003.

[45] M. Tanaka and H. Grossman, "Connexin 26 induces growth suppression, apoptosis and increased efficacy of doxorubicin in prostate cancer cells," Oncology Reports, vol. 11, no. 2, pp. 537-541, 2004.
[46] A. W. Tate, T. Lung, A. Radhakrishnan, S. D. Lim, X. Lin, and M. Edlund, "Changes in gap junctional connexin isoforms during prostate cancer progression," The Prostate, vol. 66, no. 1, pp. 19-31, 2006.

[47] Y. Yan and H. Huang, "Interplay among PI3K/AKT, PTEN/ FOXO and AR signaling in prostate cancer," Advances in Experimental Medicine \& Biology, vol. 1210, pp. 319-331, 2019.

[48] H. Chen, L. Zhou, X. Wu et al., "The PI3K/AKT pathway in the pathogenesis of prostate cancer," Frontiers in Bioscience, vol. 21, pp. 1084-1091, 2016.

[49] C. Dai, H. Heemers, and N. Sharifi, "Androgen signaling in prostate cancer," Cold Spring Harbor Perspectives in Medicine, vol. 7, no. 9, 2017.

[50] V. Murillo-Garzón and R. Kypta, "WNT signalling in prostate cancer," Nature Reviews Urology, vol. 14, no. 11, pp. 683-696, 2017.

[51] B. Y. Shorning, M. S. Dass, M. J. Smalley, and H. B. Pearson, "The PI3K-AKT-mTOR pathway and prostate cancer: at the crossroads of AR, MAPK, and WNT signaling," International Journal of Molecular Sciences, vol. 21, no. 12, 2020.

[52] T. Walle, "Methoxylated flavones, a superior cancer chemopreventive flavonoid subclass?" Seminars in Cancer Biology, vol. 17, no. 5, pp. 354-362, 2007.

[53] R. Ferraldeschi, J. Welti, J. Luo, G. Attard, and J. S. de Bono, "Targeting the androgen receptor pathway in castration-resistant prostate cancer: progresses and prospects," Oncogene, vol. 34, no. 14, pp. 1745-1757, 2015.

[54] C. Herberts, A. J. Murtha, S. Fu et al., "Activating AKT1 and PIK3CA mutations in metastatic castration-resistant prostate cancer," European Urology, vol. 78, no. 6, pp. 834-844, 2020.

[55] S. Divakar, K. Saravanan, P. Karthikeyan et al., "Iminoenamine based novel androgen receptor antagonist exhibited anti-prostate cancer activity in androgen independent prostate cancer cells through inhibition of AKT pathway," Chemico-Biological Interactions, vol. 275, pp. 22-34, 2017.

[56] W. Lim, S. Park, F. W. Bazer, and G. Song, "Naringenininduced apoptotic cell death in prostate cancer cells is mediated via the PI3K/AKT and MAPK signaling pathways," Journal of Cellular Biochemistry, vol. 118, no. 5, pp. 1118-1131, 2017. 